\title{
La incontinencia urinaria diurna al ingreso escolar es más frecuente de lo esperado
}

Daytime urinary incontinence in primary school children: A population-based survey. Sureshkumar P, Craig JC, Roy LP. J Pediatr 2000;137:814-8

\section{Objetivo}

Determinar la prevalencia, severidad y factores de riesgo de la Incontinencia Urinaria Diurna (IUD) en niños que comienzan la escuela primaria.

\section{Diseño}

Estudio poblacional de corte transversal, mediante una encuesta autoadministrada a los padres.

\section{Lugar}

Sydney, Australia.

\section{Participantes}

Se evaluó a través de sus padres, a un total de 2020 niños inscriptos para comenzar la escuela primaria, entre 4 y 6 años de edad.

\section{Evaluación de los factores de riesgo}

Los padres respondieron una encuesta que abarcaba características demográficas, status socioeconómico, antecedentes familiares y personales.

\section{Medición de resultados principales}

En la encuesta se utilizaron categorías predefinidas de severidad de IUD. Se les solicitó que registren la máxima frecuencia de IUD durante los últimos 6 meses.

\section{Resultados principales}

Respondieron 1419 familias (70\%), la edad promedio de los niños fue de 5.9 años.

El 19.2\% (IC 95\% 17.3-20.7) tuvo al menos un episodio de IUD durante los últimos 6 meses. En contraste, los maestros entrevistados reportaron sólo un 3\% de incidencia en la misma población.

\begin{tabular}{l|l|l}
\hline Incontinencia Diurna & Incidencia & Grado \\
\hline Todos los días & $0.7 \%$ & Severo \\
\hline 2 ó + veces por semana & $2.0 \%$ & Moderado \\
\hline 2 ó veces por mes & $1.5 \%$ & Leve $16.5 \%$ \\
\hline 1 vez por mes & $1.2 \%$ & \\
\hline 2 ó + veces en 6 meses & $5.0 \%$ & \\
\hline Una vez en 6 meses & $8.8 \%$ & \\
\hline Total (Al menos 1 vez en 6 meses) & $19.2 \%$ & \\
\hline
\end{tabular}

En el análisis multivariable, los únicos factores de riesgo significativos para IUD moderada o severa fueron el antecedente de un evento de estrés emocional (OR 5.7, 95\% IC 2.7-12.0), historia de IUD en hermanos varones (OR 5.3, 95\% IC 1.6-18.2) e historia de IUD en la historia familiar por parte del padre (OR 9.3, $95 \%$ IC 3.2-27.3). Sólo un $16 \%$ de los niños que padecían algún tipo de IUD habían consultado al médico, aunque ésto dependía de la severidad y en el grupo de IUD grave llegaba el $60 \%$ de consulta.

La importancia de los factores de riesgo se informa también como riesgo atribuible poblacional (exceso de "enfermedad" en la población de expuestos atribuída a la exposición); un 59\% de los casos de IUD moderados y severos pueden ser atribuidos a 3 factores de riesgo: estrés emocional $33 \%$, antecedentes en hermanos varones $8 \%$ y antecedentes paternos $18 \%$.

\section{Conclusiones}

La IUD es más frecuente durante el primer año de escolaridad que lo que se pensaba hasta ahora. El estrés emocional y la historia familiar parecen ser los principales determinantes de este trastorno.

\section{Comentario}

El control de la continencia urinaria es un proceso madurativo que presenta diferencias entre los individuos, làs familias y las culturas. Actualmente se considera normal la incontinencia urinaria diurna hasta los 4 años, luego de esa edad se recomienda estudiar a los pacientes (con una orina completa, una ecografía renal y vesical, además de los antecedentes y el examen físico).1-3

A diferencia de la enuresis nocturna, en la diurna no hay hasta ahora datos fehacientes de su frecuencia ni su asociación con factores de riesgo.

Este estudio es apropiado para conocer la incidencia de IUD en los últimos 6 meses e involucra una gran cantidad de niños tomados al azar. La tasa de respuesta fue buena $(70 \%)$, pero con la posible incorporación del sesgo de reporte (tener un hijo con IUD modificaría la probabilidad de contestar la encuesta).

La población australiana probablemente no sea muy diferente de la nuestra como para no poder aplicar estos resultados en nuestra práctica, por lo tanto la IUD parece ser más frecuente de lo esperado, presentándose por ejemplo en 1 cada 20 niños (5.4\%). Para evaluar causalidad el diseño ideal seria un ensayo aleatorizado, pero es impracticable con la IUD. No obstante en un estudio prospectivo se podrían medir los factores de riesgo y monitorear la aparición del evento en el tiempo, y a diferencia de este trabajo (estudio transversal) no requiere que los familiares recuerden la aparición del evento, los antecedentes familiares y la exposición a estrés, evitando el posible sesgo de recuerdo que podría estar afectando los resultados.

Los autores reconocen como una limitación del estudio el hecho de no poder obtener un urocultivo para descartar infección urinaria en los casos de IUD, la identificación de estos casos disminuiría la importancia de los factores de riesgo detectados. Por otro lado, existe una concordancia entre estos factores de riesgo y los que ya se conocen para la incontinencia urinaria nocturna, lo que habla a favor de la asociación.

Tomando en cuenta estos datos, aunque no se pueda asumir como definitivo, los antecedentes en el padre y hermanos y experiencias de estrés emocional tienen un papel importante en el desarrollo de IUD.

La importancia de los antecedentes familiares tal vez explique el motivo de la baja tasa de consulta, ya que muchas familias no asuman como "enfermedad" que un niño tenga IUD pasando los 5 años ya que su padre o sus hermanos han pasado por lo mismo, siendo un trastorno autolimitado en la gran mayoría de los pacientes.

\section{Dr. Alejandro Lopez Osornio [ Unidad de Medicina Familiar y Preventiva. Hospital Italiano de Buenos Aires ]}

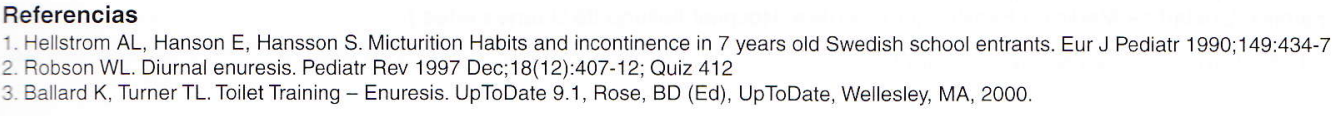

